\title{
Research on Overload Monitoring and Alarming based on APP
}

\author{
Feng Pengfei $^{* 1,2}$, Jin Huiqing ${ }^{1,2}$, Gu Jiangyang ${ }^{1}$ and Han Jian $^{3}$ \\ ${ }^{1}$ Anhui Sanlian University \\ ${ }^{2}$ National Vehicle Driving Safety Engineering Technology Research Center \\ ${ }_{3}^{3}$ Jianghuai Automobile Technology Center, Hefei, 230601 \\ 18063780@qq.com
}

\begin{abstract}
As the acceleration of urban construction, the dump heavies (building soil-transporting vehicle) shuttle back and forth in urban road. For the sake of profits, the crazy overload transportation makes the vehicles lose braking control and steering ability in emergency state. The common occurrence of traffic disasters brings huge hidden troable of urban traffic safety. Its harm is self-evident, causing serious domage to infrastructure, to threaten the safety of life and property of the people, to dis rupt the marke economic order, but also cause environmental pollution. In recent years, the traffic management departments and urban management departments jointly monitor the discharging heavy trucks in the forms of surveillance and reporting by the masses, installing camera and monitor, self-discipline and self-supervision of investors, eter These forms are limited to the forms and surface, so they cannot effectively stop the overload of self-discharging trucks. How to better and accurately monitor and stop the over-load? The paper intends to achieve intelligent monitoring and alarming an theverload of dump heavy through internet + public transportation monitoring. First the controller is selected and the weighting sensing system is deslghed through selecting the weighting sensor and determining the installation position of weighting sensor. Then the construction of APP overload monitoring is finished by the three steps including constructing dual-core system, generating database and designing APR display module. Finally after finishing the design of intelligent overload detection system, the paper conducts experiments to validate its effectiveness. The results show that when considering no electromagnetic, the overload equipment and $A P R$ software system could receive the overload information timely and reliably.
\end{abstract}

Keywords: Road Transportation; APP Software; Overload Monitoring; Dump heavy

\section{Introduction}

In recentyears, as the acceleration of urban construction in China and the promotion of urban-rural integration process, dump heavy (generally called "building soil-transporting vehicle becomes an essential forces in urban construction. The busy dump heavies shintle in the city carrying muck or building materials. However, the transportation safety of the vehicles makes the people anxious and uneasy. The malignant traffic accidents occur sometimes, such as people is rolled by soil-transporting vehicles. According to the incomplete statistics of Legal Daily, the media reported over 40 soil-transporting vehicle accidents in half a month in October 2014 [1]. The reason is that nearly 70\% accidents are caused by vehicle overload, brake failure or decrease of brake efficiency. One more ton vehicles are overloaded, the brake distance will extend one more meter. Overload makes the tire pressure greater than the rated load and easy to cause a blowout.

"Overload" has become the defaulted "guild regulations". The drivers of dump heavy have objective and subjective causes for overload. Driven by huge economic benefits, some drivers of dump heavy ignore the bearing limit of vehicle with fluke mind, so that 
the overload phenomena increasingly intensify and exist. Harm of the problem is self-evident, causing serious damage to infrastructure, to threaten the safety of life and property of the people, to disrupt the market economic order, but also cause environmental pollution.

How to effectively stop the overload phenomenon of dump heavy? In most cities, the traffic management department and urban management department jointly monitor by setting up the organization of overload detection and management or the masses monitor the phenomena. The management people detect whether the dump heavy is overloaded by first stopping the vehicle. And this will lead to traffic jams and very low efficiency. After knowing the detection positions and other relevant detection information, drivers would drive around to avoid detection. On the other hand, results of artificial monitoring are subjective and the accuracy is low, bribery phenomenon is also easy to happen. So these forms just stay in surface and fail to effectively stop the overload of self-discharging trucks.

Some innovative practices are taken in some cities, such as installing camera on dump heavy in Fuzhou; taking GPS to position and monitor the illegal behaviors of dump heavy. But these methods are hard to monitor the overload of heavy truck. In Xi' an the investors bear the joint liability, while the mode of self-discipline and self-monitoring mode achieves unobvious effect.

Overload detection is on the premise of vehicle weighing. Traditional weighing is conducted mainly through weighbridge and dynamic yeighing stations, while these two weighting tools could not cover the entire road network, so their application is limited. At present, the method widely used in vehicle ovelload recognition is weighing platform measurement. Weighing platform measurement method is divided into the static measurement method and the dynamic measurement method. The static weight measurement has been basically eliminated at oresent, although it could achieve great measurement accuracy, because the yehicles have to stop and wait in line for detection and this would easily make the traific jam; The dynamic weighting measurement method could be divided into culvent type dynamic weighting system, bridge dynamic measurement system and axle test dymanic weighing system according to the detection principle. While the dyamic weighting measurement system has greatly improvement in the measurement efficiency, but the measurement accuracy needs to be improved. But both static weighting measurement system and the dynamic weighting measurement system require vehicles to stay on specific weighing platform, and the weighting could be only finished in particular cases, unable to monitor whether the vehicle is overloaded anytime and anywhere intelligently.

In recent years, the concept of "Internet + " is put forward and have been widely applied to all walks of life and caused so many industry changes. Relying on the Internet information technology, "Internet $+"$ could achieve the combination of the Internet and traditional industries and reconstruct business model to complete the economic transformation and upgrade by optimizing the factors of production and updating the busines system. "Internet + " program aims to give full play to the advantages of the internet, to realize the in-depth integration of Internet and traditional industries, to promote economic productivity through industrial upgrading, and finally to realize the increase of social wealth. In the field of public transportation, "Internet +" has made a lot of progress including Intelligent transportation scheduling system, car networking system and autopilot system and so on.

The paper intends to achieve intelligent monitoring and alarm on the overload of dump heavy through internet + public transportation monitoring, in order to facilitate the urban traffic safety management. First the controller is selected and the weighting sensing system is designed. Then the construction of APP overload monitoring is finished. Finally after finishing the design of intelligent overload detection system, the paper conducts experiments to validate its effectiveness. 


\section{Overload Monitoring of Dump Heavy}

\subsection{Selection of Controller}

The intelligent control of overload of dump heavy shall give priority to hardware firstly in order to make proactive effect in the actual work. According to the existing intelligent control standards and the actual allowable standards in the world, the design ideology of controller shall consider from three aspects: firstly, model MSP430F149 SCM is selected to guarantee minimum energy consumption and maintain the good effect in the actual operation process. The model may execute one command within 1 clock cycle. Upon the corresponding test, the command speed may reach 8MIPS and completely meet the intelligent control requirements for overload of dump heavy. Secondly, considering a large amount of information will be changed into digital information from A/D analog-digital conversion module and the layout of overall CAN line, we will use 16-bit $\mathrm{A} / \mathrm{D}$ converter, and the model is AD7705B [2]. It is a signal converter developed and researched based on CAN bus. It is suitable to the design with outstanding efficiency. Thirdly, in the operation of controller, during the loading process of dump heavy, the truck quality may be effectively measured through weighing the senso . The controller will generate weaker voltage signal and the signal will be converted to digital signal through a series of treatment of various hardware equipment and be sent to the collection device. In case of overload, LCD screen will display the relevan information and the workers will mandatorily implement overload control measures. If not, LCD screen will display the existing information to prevent overload in the subsequent transportation, thus the intelligent control is achieved in a real sense.

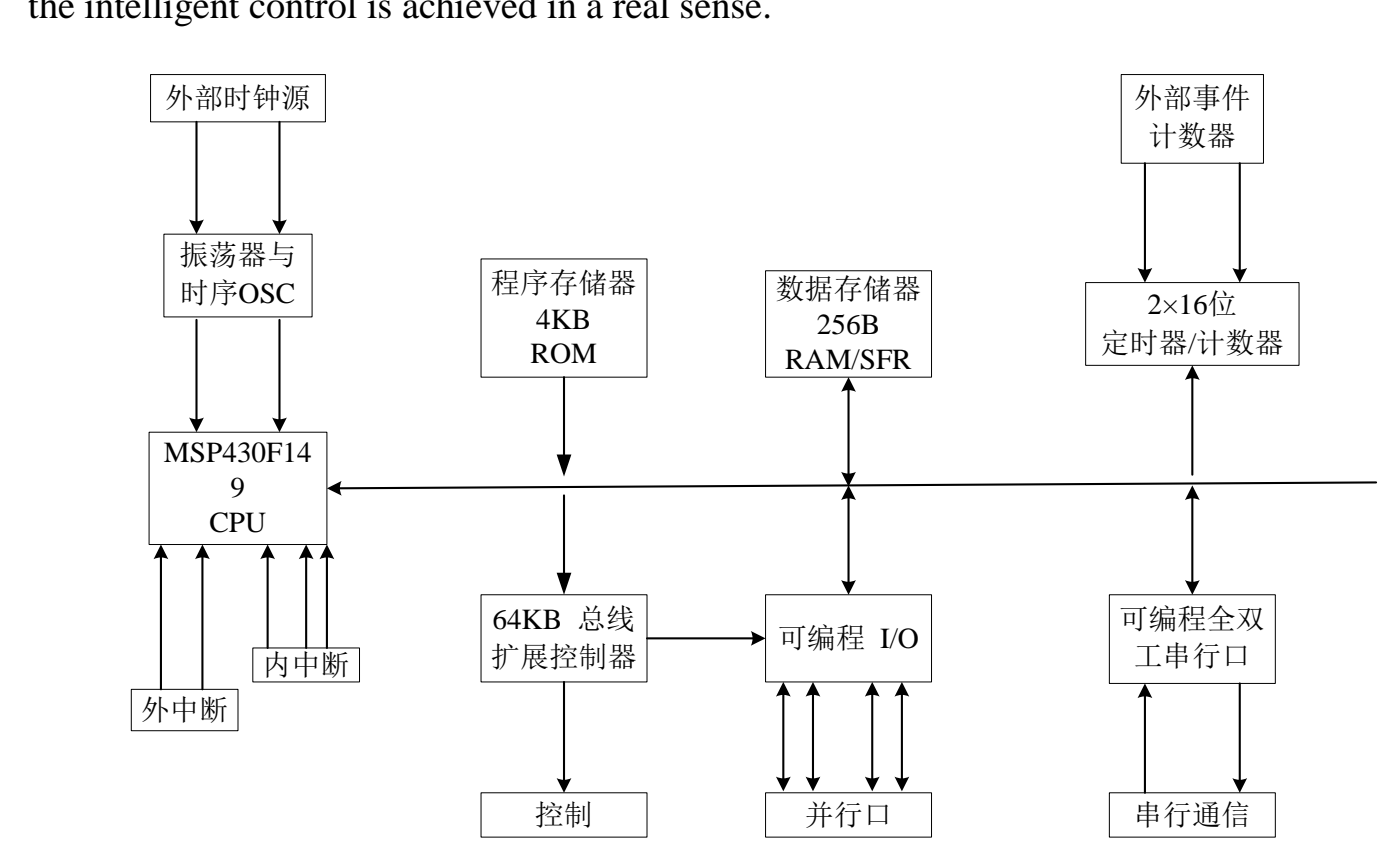

Figure 1. MSP430F149 Monolithic Block Diagram

\subsection{Design of Weighing Sensing System}

\subsubsection{Selection of Weighing Sensor}

In order to realize the intelligent monitoring and alarming, selection of weighting sensor, as the basic component, is the first key issue to be solved. And the selection should meet the following requirement. The intelligent control of overload of dump heavy 
shall guarantee that it may not be changed by the drivers and be effective for long term, so resistance strain weighing sensor is used. In the aspect of monitoring quality principle, the detected quality may be converted to the power signal used by the system and the signal may be pasted on the elastic and sensitive element, and then form bridge in a proper mode. The real-time monitoring may be achieved when meeting bridge balance conditions:

$$
(R+\Delta R)^{2}=(R-\Delta R)^{2}
$$

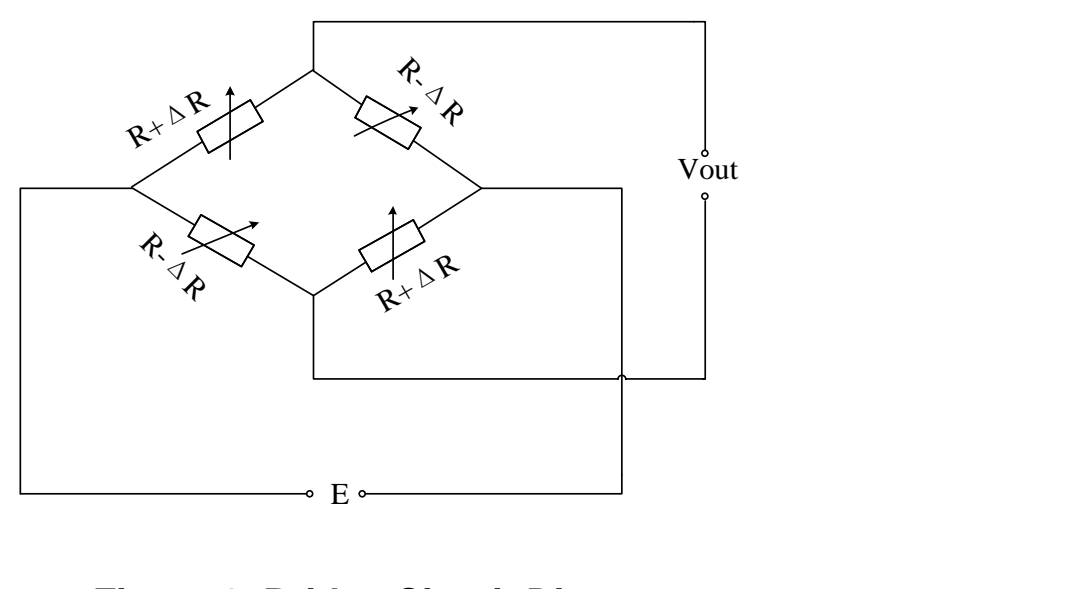

Figure 2. Bridge Circuit Diagram

From the internal composition, resistance strain weighing sensor mainly consists of two parts and each part finishes different works. The first part is elastic sensitive element, and its main function is to finish the measurement of the quantity to the strain of elastic body. This step is of great importance because it is the original and basic input of the entire module and its accuracy determines that of the entire system; the second part is the resistance strain piece of sensor element, whose main work is converting the strain of elastic body to resistance and then output the electric data, thus the load information could be displayed digitatly and the collection of basic data could be completed. According to the design requirements, AD-S321 module products are suitable to be used here because the product sufficiently uses the working mode of resistance strain weighing sensor [3-4].

In order to neet the atual requirements, referring to the national metering and metrological verification regulations, the force standard machine with $20 \%$ load ability is used to conduct conventional test and take records and statistics in the test process, in order to determine the error scope of the module and the calibration of sensitivity, furthermore to provide experimental support to illustrate that the module is able to complete the weighing sensor work and achieve our targets. The experimental data are shown in Table 1 . 
Table 1. Calibration of Temperature Load Test Data Tables

\begin{tabular}{|c|c|c|c|c|c|}
\hline Load contrast/t & $\begin{array}{c}\text { Average } \\
\text { Initial 20 }\end{array}$ & $\begin{array}{l}\text { Repeated } \\
\text { error }\end{array}$ & $\begin{array}{c}\text { Load } \\
\text { contrast/t }\end{array}$ & $\begin{array}{l}\text { Average High } \\
\text { temperature } 40\end{array}$ & $\begin{array}{l}\text { Repeated } \\
\text { error }\end{array}$ \\
\hline 0 & 0.000 & 0 & 0 & 0.000 & 0 \\
\hline 1 & 0.998 & 2 & 1 & 0.999 & 2 \\
\hline 2.5 & 2.498 & 4 & 2.5 & 2.499 & 2 \\
\hline 5 & 4.997 & 3 & 5 & 4.999 & 2 \\
\hline 10 & 9.999 & 3 & 10 & 9.999 & \\
\hline 15 & 15.002 & 5 & 15 & 15.000 & 2 \\
\hline 20 & 20.003 & 4 & 20 & ת ח & 3 \\
\hline Load contrast/t & $\begin{array}{c}\text { Average } \\
\text { Low } \\
\text { temperature } \\
-10\end{array}$ & $\begin{array}{l}\text { Repeated } \\
\text { error }\end{array}$ & & $\begin{array}{l}\text { Termination } \\
\text { temperature } 20\end{array}$ & $\begin{array}{l}\text { Repeated } \\
\text { error }\end{array}$ \\
\hline 0 & 0.000 & & 0 & 0.000 & 0 \\
\hline 1 & 1.000 & & & 0.999 & 1 \\
\hline 2.5 & 2.500 & & .5 & 2.499 & 1 \\
\hline 5 & 5.002 & & 5 & 5.000 & 2 \\
\hline 10 & 10.004 & & 10 & 10.000 & 2 \\
\hline 15 & 12 & & 15 & 15.002 & 2 \\
\hline 20 & 010 & 2 & 20 & 20.004 & 3 \\
\hline
\end{tabular}

Note: The serles of test is conducted with three-time meaning method.

The above is the organization of all data. The below is the chart of the repeated error of the module.

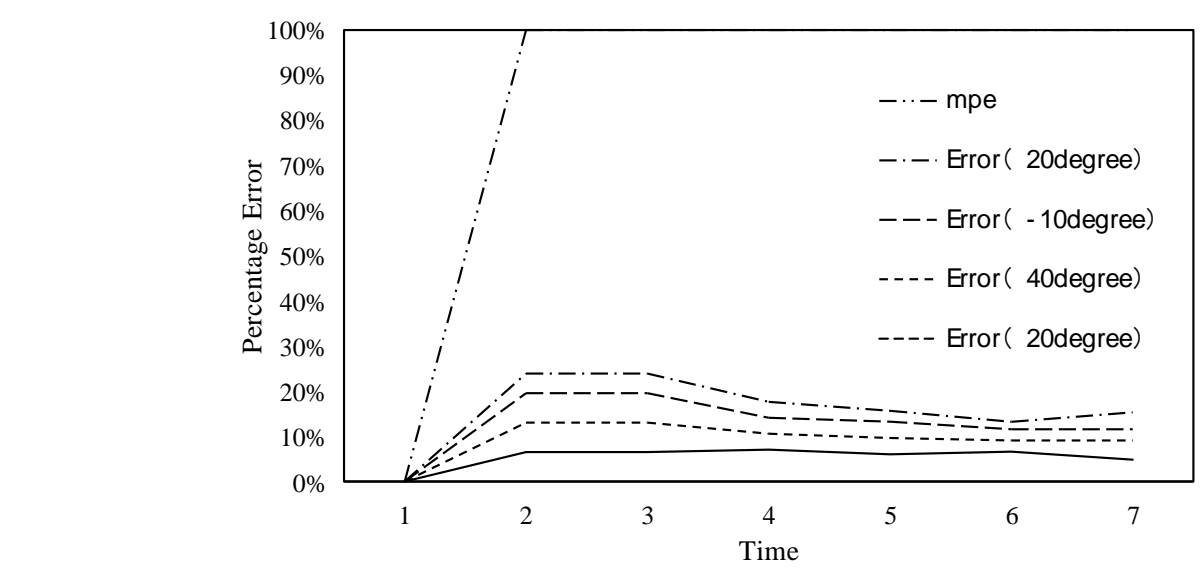

Figure 3. Repeated Error Area Chart 
As it is shown in Figure 3, under the national standard conditions, the module is far less than the maximum allowable repeated error. The calculation chart of weighing error of the module is shown below.

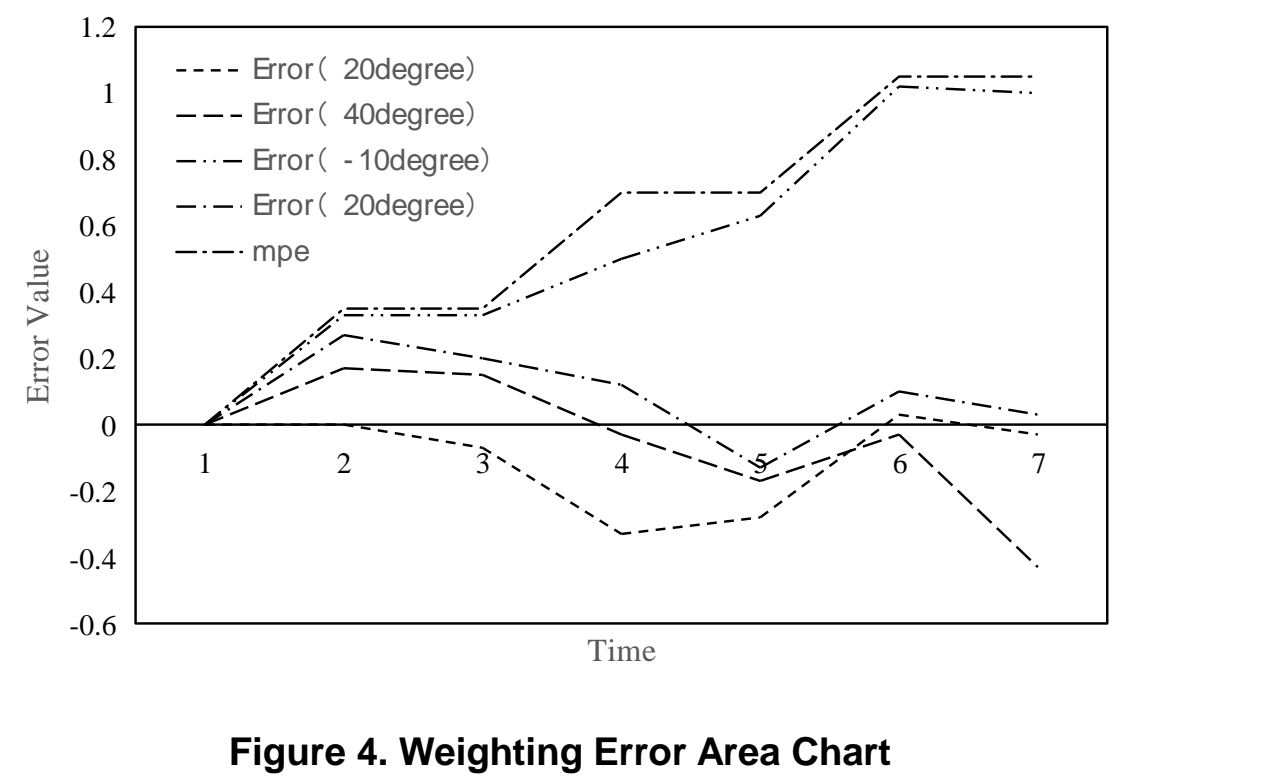

As shown in Figure 4, under the national standard conditions, if the weighing data of the selected module is lower than the maximum allowable error, which may ensure the accuracy of weighing date. It is shown that the error of resistance strain weighing sensor is less than the maximum allowable error, namely it is negligible. Therefore, the module may achieve ideal intelligent control effect with good performance.

\subsubsection{Determination of Installation Position of Weighing Sensor}

To determine the installation position of sensors is another key problem to achieve intelligent overload monitoring andalarming. As an important device of overload signals, it inappropriate installation position of sensor could lead to error detection results, so the correct installation of senser is the first step to insure the effect of test. The sensor shall meet the dynamic monitoring of dump heavy. The driving system of dump heavy mainly is non-independent suspension system, so the installation position of weighing sensor is determined after measurement. In heavy truck series autos, in order to distribute the quality on rack evenly, a kind of elastic element used most is plate spring. The plate sprint is an elastic beam with similar strength composed by several alloy spring pieces with equal width but different length. [5] 


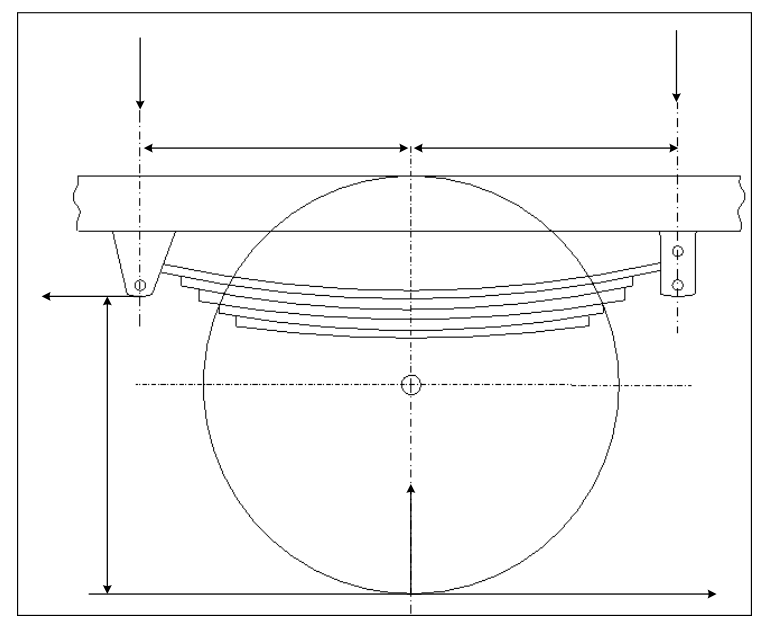

Figure 5. Steel Plate Spring

As shown in Figure 5, when the vehicle is driving under load, the plate spring will suffer from vertical load and the leaf spring will be deformed under force and tend to concave upward. At the time, the bridge and rack will draw closer, and the vehicle weight will be distributed evenly. The load acting on the plate spring is:

$$
\begin{aligned}
& P_{1}=\frac{m_{1} G_{1}}{L_{1}+L_{2}}\left(L_{2}-\varphi c\right) \\
& P_{2}=\frac{m_{1} G_{1}}{L_{1}-L_{2}}\left(L_{1}+\varphi c\right)
\end{aligned}
$$

Where, $P_{1}$ and $P_{2}$ is the frontand back load of plate spring; $G_{1}$ is the load acting on wheel; $C$ is the distance from fixed point of plate spring to pavement; $L_{1}$ and $L_{2}$ is the front and back length of plate spring; $\varphi \square$ is the coefficient of road adhesion; ${ }^{m_{1}}$ is load distribution coefficient of front axis.

To make the torque balanced Suppose the load point is respectively in " $A$ " and " $B$ " in Figure 5, namely the weighing sensor is installed in " $\mathrm{A}$ " and " $\mathrm{B}$ ". The monitoring information of yehicle lôad is the most accurate.

To verify whether the in tallation position is in "A" and "B" in Figure 5, we divide the area of plate spring, byilge and rack to "1" (between ridge and plate spring), "2" (between plate springs"), " 3 " (bridge near to the plate spring), "4" (below plate spring) and "5" (rack near to plate spring), and then conduct overload test. In this way, we may test which part is closest to the actual load. Thus, the installation position may be determined. Upon test, we may draw the load comparison curve 6 of 5 areas. Upon analysis, we may see that the error in " 1 " and " 3 " is smaller in neutral test. " 1 " fits well with the actual load quantity, sothe installation position of sensor is in " $A$ " and " $B$ " in area " 1 ". 


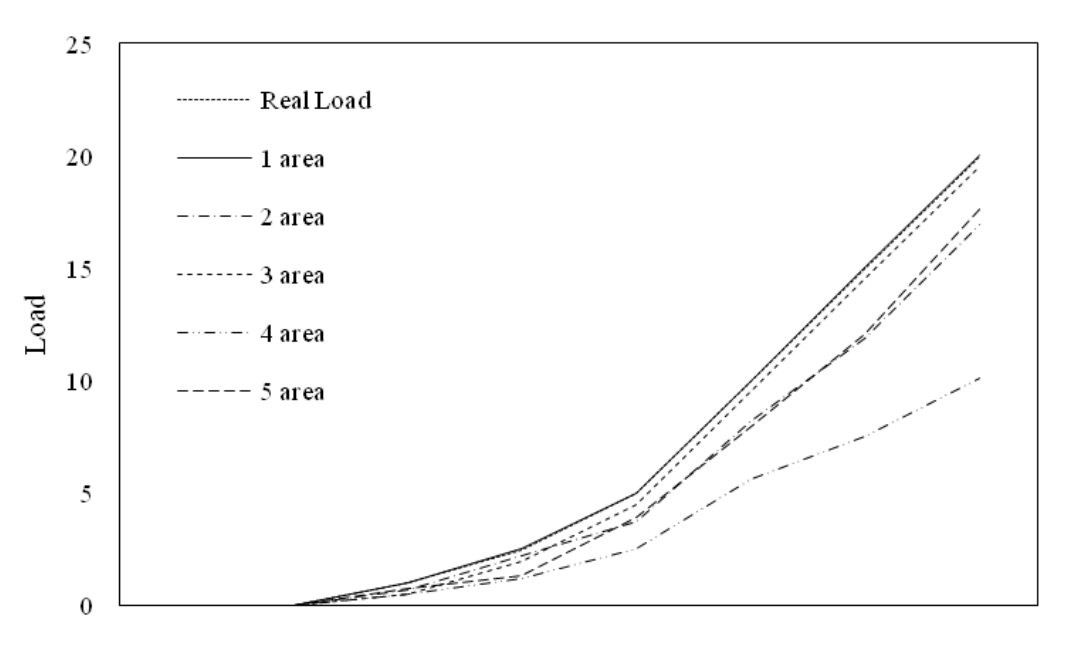

Figure 6. Five Regional Load Contrast Figure

\section{Construction of APP Overload Monitoring}

After determining the used hardware of detection system, the hext work is to finish the soft part of the system and construct APP overload monitoring. This work includes three main tasks. That is construction of dual-core system, generation of database and design of APP display module, which are introduced separately as follows:

\subsection{Construction of Dual-Core Systenn}

The construction of dual-core nodel system is shown in Figure 7. One core takes use of APP platform to integrate four functions: reborting, receiving reporting, monitoring and punishing. It will save monitormg punishment time to the traffic regulators and make effective punishment judgment to the subjective overload behavior of dump heavy drivers.

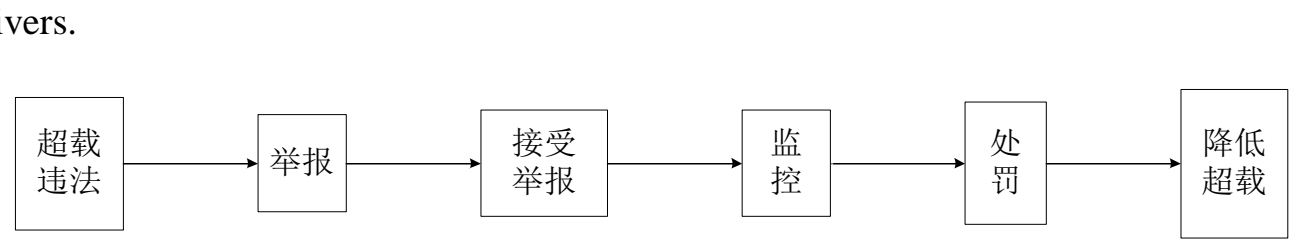

\section{Figure 7. Dump Heavy Progressive Overload Law Enforcement Procedure \\ Chart}

Another core is the overload behavior monitoring of dump heavy drivers based on the intelligence of MSP430F149 SCM [7]. It may achieve pre-warning of overload vehicle, GPS position monitoring and vehicle owner information monitoring through real-time positioning, point penalty and other monitoring functions. Figure 8 is the block diagram of overload real-time monitoring based on APP software. MSP430F149 SCM is installed on the vehicle to dynamically analyze and judge the overload conditions and send the monitoring data to mobile terminal APP software. 


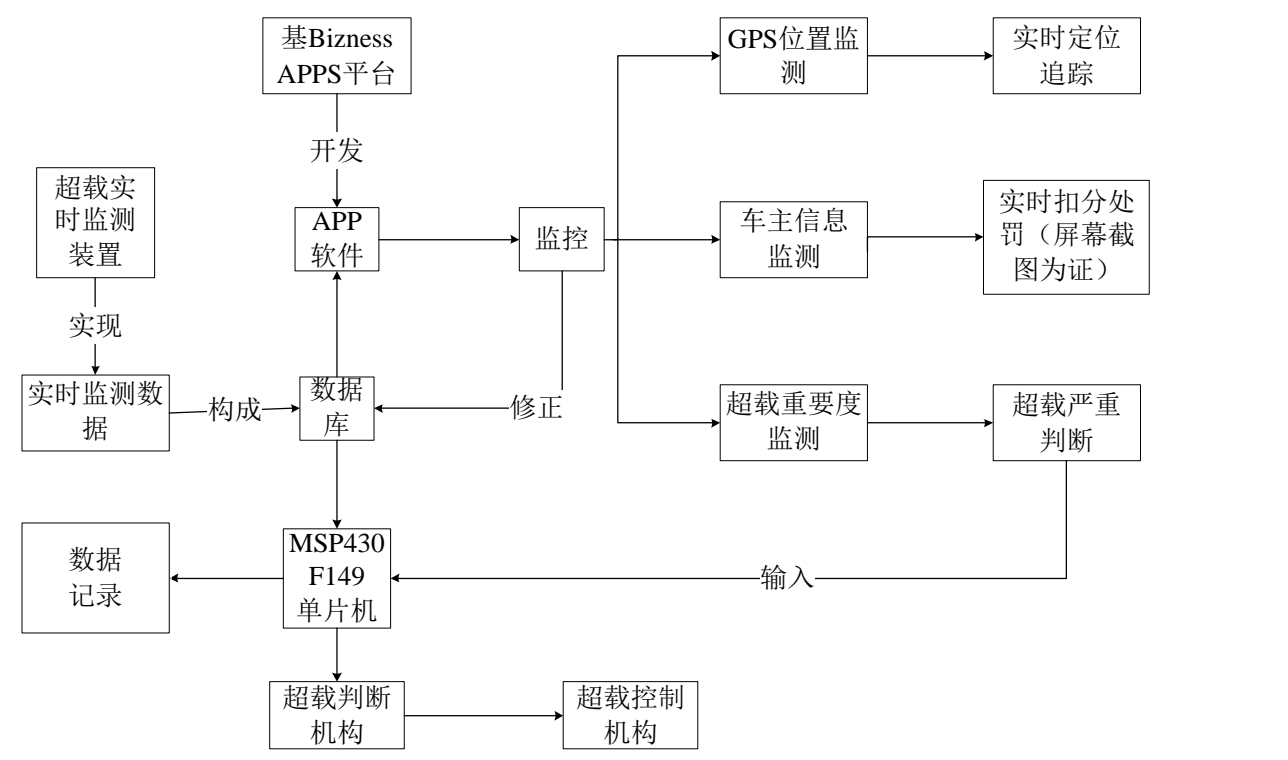

Figure 8. Overload Real-Time Monitoring Framework Model Básed on APP

\subsection{Generation of Database}

Database stores data in a certain way together with as littie redundancy as possible for multiple users to share, and it is independent with the application of data collection and provides powerful information support for the application software. So database is very important and indispensable componentol APP.

Take Navicat for MySQL as the platform to build a database and make the database structured. The data in the database shall be updated in real time. The database source is shown in Figure 9.

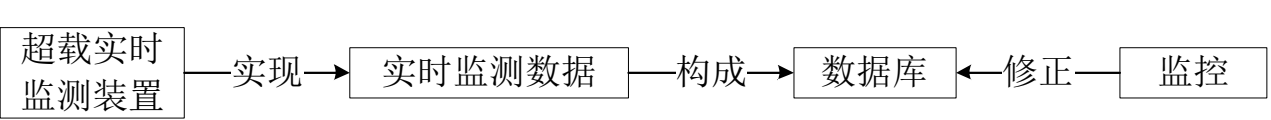

\section{Figure 9. Figure Database Data Sources}

The data updating comes from two aspects: one is the basic data composed by real-time monitoring data fromoveload monitoring; the other is the revised data fed after APP software operation. For example, if the vehicle with plate of "WAN AXXXXX" has been punished, the original monitoring data "vehicle overload $3 \mathrm{X} \%$ " will be changed to "vehicle overload has been processed", in order to update and correct the database. In order to make-the traffic regulators make statistics effectively, the database background treatment rights will be delivered to the traffic law department.

\subsection{APP Display Module Design}

App display module is mainly responsible to present the results of system processing for the users, and its function is a bridge connecting the App and user. The performance of it will directly affect the user's experience of the App. Here three main aspects of the display are designed and introduced separately, namely distance display, vehicle owner information display and position display.

\subsubsection{Distance Display}

The traditional computer intervention or information warning mode goes against the practical operation with high cost. the design creates a kind of mobile user platform 
specified used in traffic regulation department integrating warning information report, overload automatic alarming, overload information automatic renewal on Navicat for MySQL software platform. Based on the big data conditions of database, monitor and punish the drivers of dump heavy suffering from subjective overload or artificial damages of overload equipment. The integrated application of the internet and GPS is the important support of the APP. High-speed mobile internet and accurate GPS positioning may achieve accurate real-time monitoring and alarming functions. The equipment number of truck installing monitoring equipment will be set as the plate of the vehicle. The load of the vehicle may be monitored online. Within the scope of $30 \mathrm{~km}$, if the mobile user logs in the monitoring APP software, he/she may receive the load vehicle information.

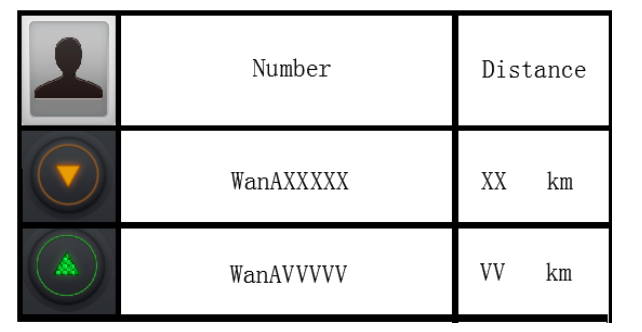

Figure 10. Schematic Diagram of Real-fime Display Module

\subsubsection{Vehicle Owner Information Display}

In order to display the overload vehicle information and vehicle owner's information in details, the traffic regulators may use the special account number to log in APP. After logging in, "logging" will be displayed in the top lent as shown in Figure 10. When the vehicle with plate "WAN AXXXXX" overloads, the notice bar in the left will display "yellow and downward triangle" pattern and the distance bar will display "XXKm". When the vehicle with plate "WAN AXXXXX" does not overload, it will display "green and upward triangle". ClCk the "number" of overload vehicle to the secondary interface displaying the vehicle owner information to display the overload information and vehicle owner's information.

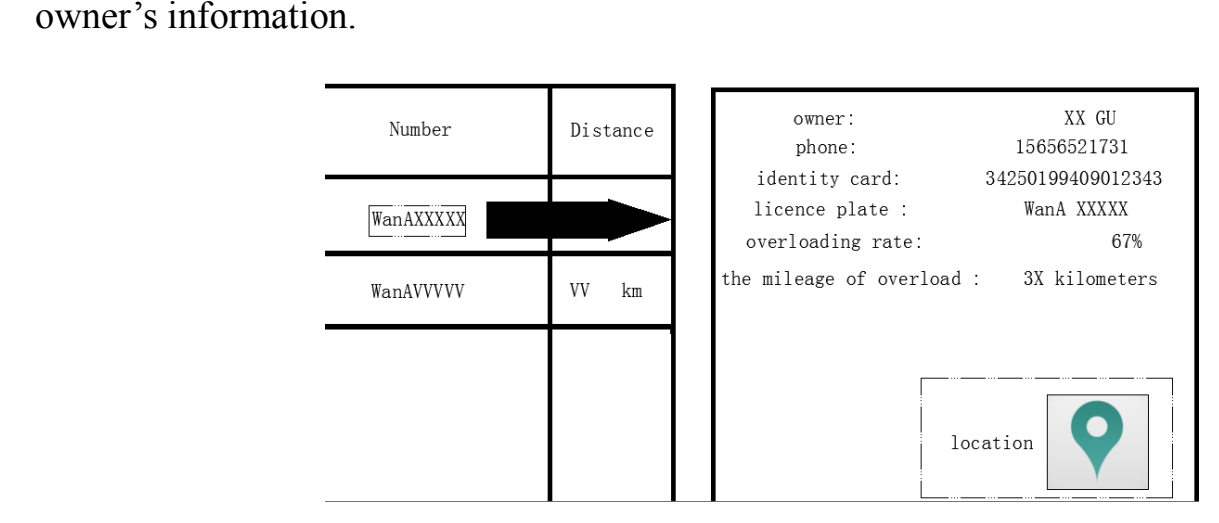

Figure 11. Overload Information Automatic Generation Module

\subsubsection{Position display}

After determining the overload information and vehicle owner information, we may further track the position information of the overload vehicle. As shown in Figure 11, the red circle in the bottom right displays the three-level menu of "position information", which may be linked to the mobile phone map and display the vehicle's position information. The overload vehicle is set as the end point and the top right will display 
"WAN AXXXXX" and real-time speed "3XKm". In such way, the traffic managers are convenient to regulate the overload vehicle.

\section{APP Software Acceptance Test}

\subsection{Preconditions and Target of Test}

After the design of intelligent overload detection system, the paper conducts experiments to validate its effectiveness. Experimental conditions mainly consider the weather factors and different network environment factor. The weather mainly divides into sunny days and rainy days, and the network conditions mainly divide into WIFI, 4G, $3 \mathrm{G}$ and $2 \mathrm{G}$. The overload amount is set as $10 \%$, and the overload vehicle distance is within $30 \mathrm{Km}$. Specific test conditions are shown as follows:

Table 2. Specific Test Conditions

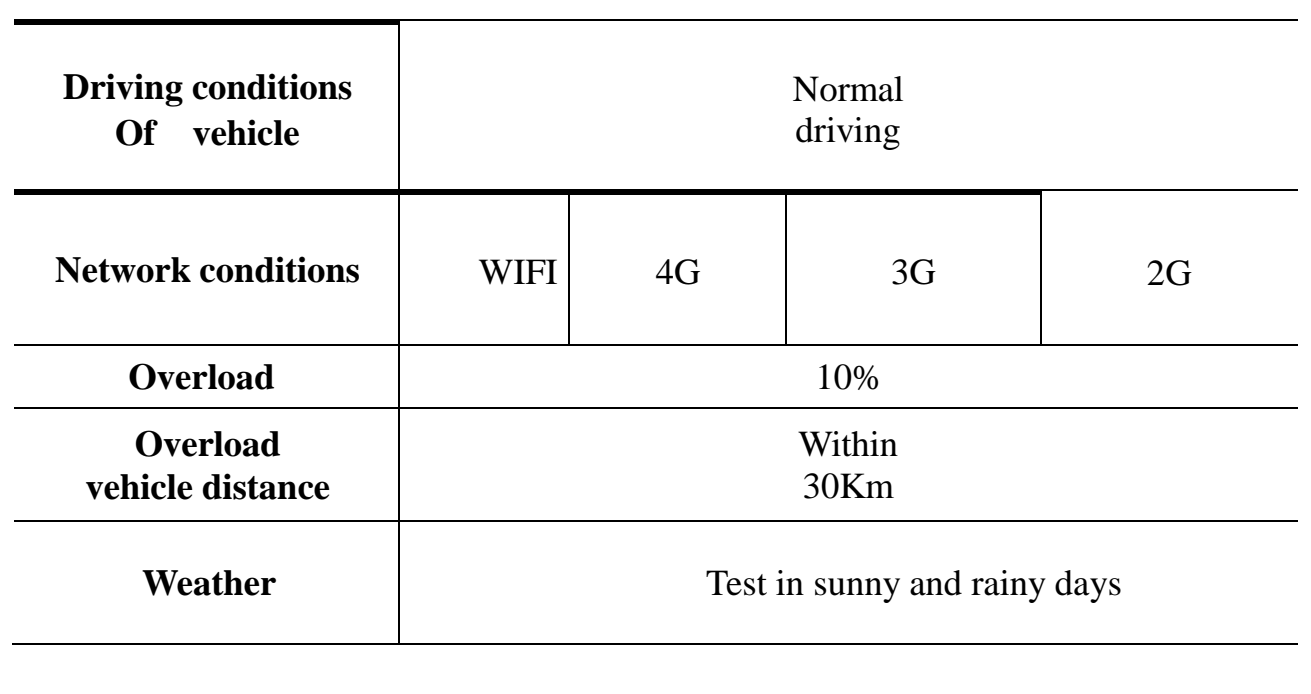

Test target could be described as follows: under the mobile network conditions of $2 \mathrm{G}$ and above, the vehicle overload may reach $10 \%$. If the overload vehicle is within $30 \mathrm{Km}$, the overload mas be detected within 60 s in normal driving conditions, sunny and rainy days and real-time monitoring may be conducted through mobile APP.

\subsection{Test Results}

After conducting the experiments under different conditions, the test results are obtained and could be described as follows: in sunny days, the real-time data may be monitored within 60s in the network environment except that 5 minutes are needed in $2 \mathrm{G}$ network In rainy days, the data cannot be detected in $2 \mathrm{G}$ network and may be detected within 2 minutes in $3 \mathrm{G}$ network, and within 60 s in other network environment. 


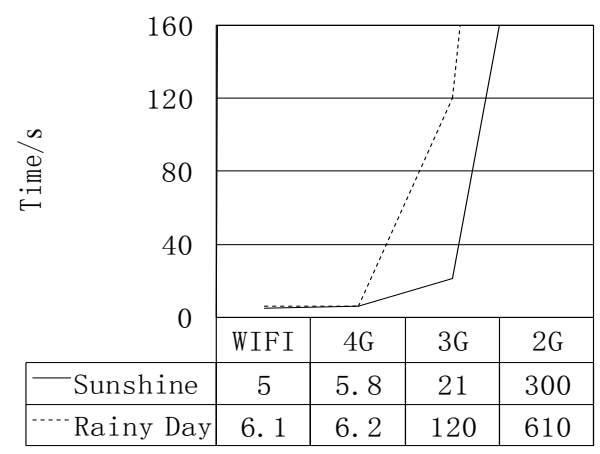

Figure 12. APP Software Receiver Test

When considering no electromagnetic, the overload equipment and APP software system may receive the overload information timely and reliably. It is recommended to monitor in WIFI and 4G mobile internet environmen to achieve ideal effect. So the designed intelligent overload monitoring and alarning system based on APP could meet our requirement greatly.

\section{Summary and Outlook}

In order to realize the overload monitoring and alaming intelligently, the paper makes full use of the concept and theory of "Internet "and takes dump heavy vehicle as the research sample, analyzes the selectron of overload monitoring system controller, design of weighing sensor system and development of software, achieves overload real-time monitoring of mobile internet combining with internet APP functions, and conducts feasibility analysis and design of display module and information processing module. Upon test, the real-time monitoring effect to the overload dump heavy is good if there is mobile internet. The research not only deters the overload executers, but also facilitates the traffic safety management and punishment of urban traffic management department and law enforcement department. The overload transportation of passenger car is also the potential traffic danger in ourcountry, which is especially serious in festivals and holidays, while different from dump heavy, the passenger transportation is characterized by randomness and renote distance. It is anticipated to obtain the further research achievements in passenger transport overload on the basis of the research.

\section{References}

[1] "Why three department joint are enforcement control "crazy" residue in car", Jurisprudence Daily, (2014)

2] S. A. Li, "16-bit 20ms/s sectional current steering structure design of CMOS D/A converter", Xian university of electronic science and technology master's thesis, (2013).

[3] Q. Wang, "Resistance strain type weighing sensor is designed", Journal wood processing machinery, vol. 3 , no. 51, (2005).

[4] G. Z. Zhou, L. C. Ma and M. L. Liu, "Motor overload intelligent controller on vehicle dynamic weighing system research", Journal Agricultural equipment and vehicle engineering, vol. 11, no. 587, (2012).

[5] S. T. Zhou, H. W. Huang and J. Z. Fu, "Based on contact friction of variable cross-section leaf spring suspension performance analysis", Journal of human university, vol. 5, no. 153, (2013).

[6] J. Dong, J. H. Ma and Z. X. Wang, "A general automobile instrument signal converter design", Journal Electronics world, vol. 3, no. 231, (2013).

[7] Q. M. Cheng, G. T. Zhang and L. Z. Wang, "Based on the AHP evaluation index system of urban road traffic safety", Journal Transportation science and economy, vol. 5, no. 173, (2014).

[8] Q. K. Cheng, L. C. Na and H. Huo, "Excellence engineers education oriented database technique 


\section{Authors}

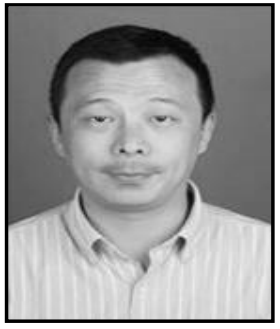

Feng Pengfei, He is a lecturer of National Vehicle Driving Safety Engineering Technology Research Center in Anhui Sanlian University. His Email address is fengpengfei@126.com. His communication address is No. 47, Hean Road, Hefei City Economic and Technical Development Zone.

Jin Huiqing, His is a professor of National Vehicle Driving Safety Engineering Technology Research Center of Anhui Sanlian University. His communication address is No. 47, Hean Road, Hefei City Economic and Technical Development Zone.

Gu Jiangyang, He is a student of Sanlian University, and his compunication address is No. 47, Hean Road, Hefei City Economic and Technical Developmentzone. His Email is943525232@qq.com.

Han Jian, He is an engineer of Jianghuai automobile technology center test department, his communication address is No. 99, Ziyun Road, Shushan District, Hefei City, Anhui Province. His Email is hjian0819@126.com

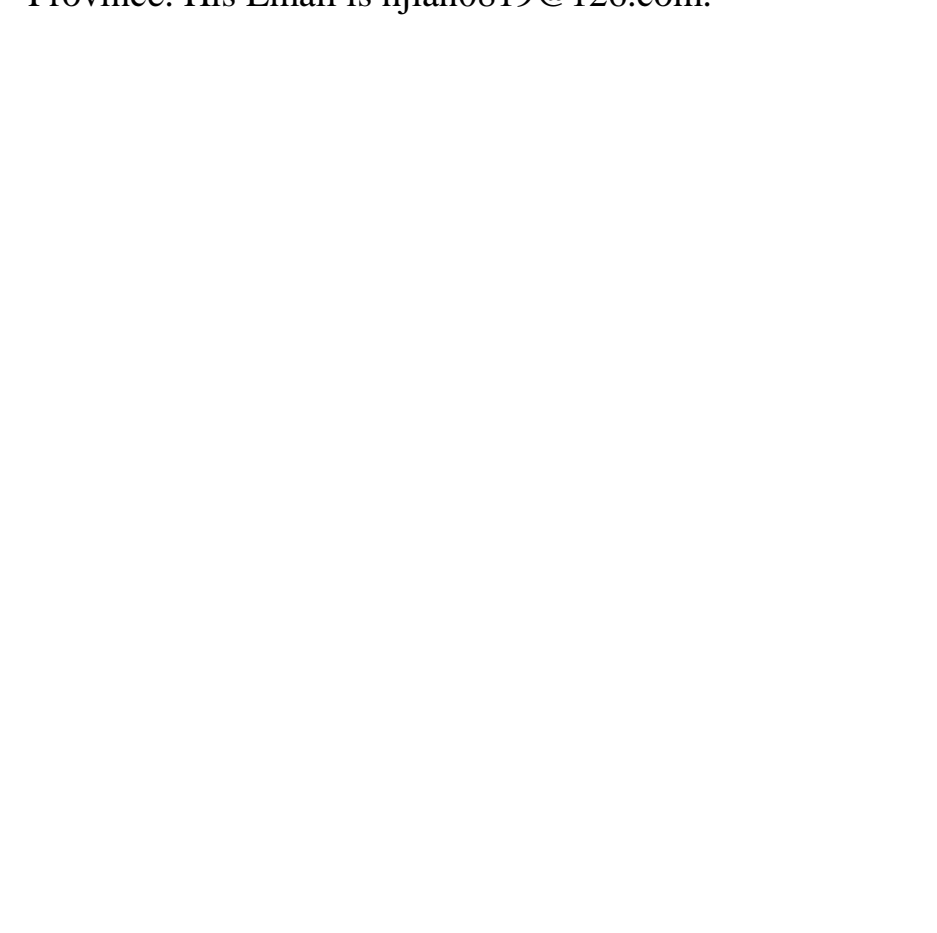


International Journal of Multimedia and Ubiquitous Engineering

Vol.11, No.11 (2016)

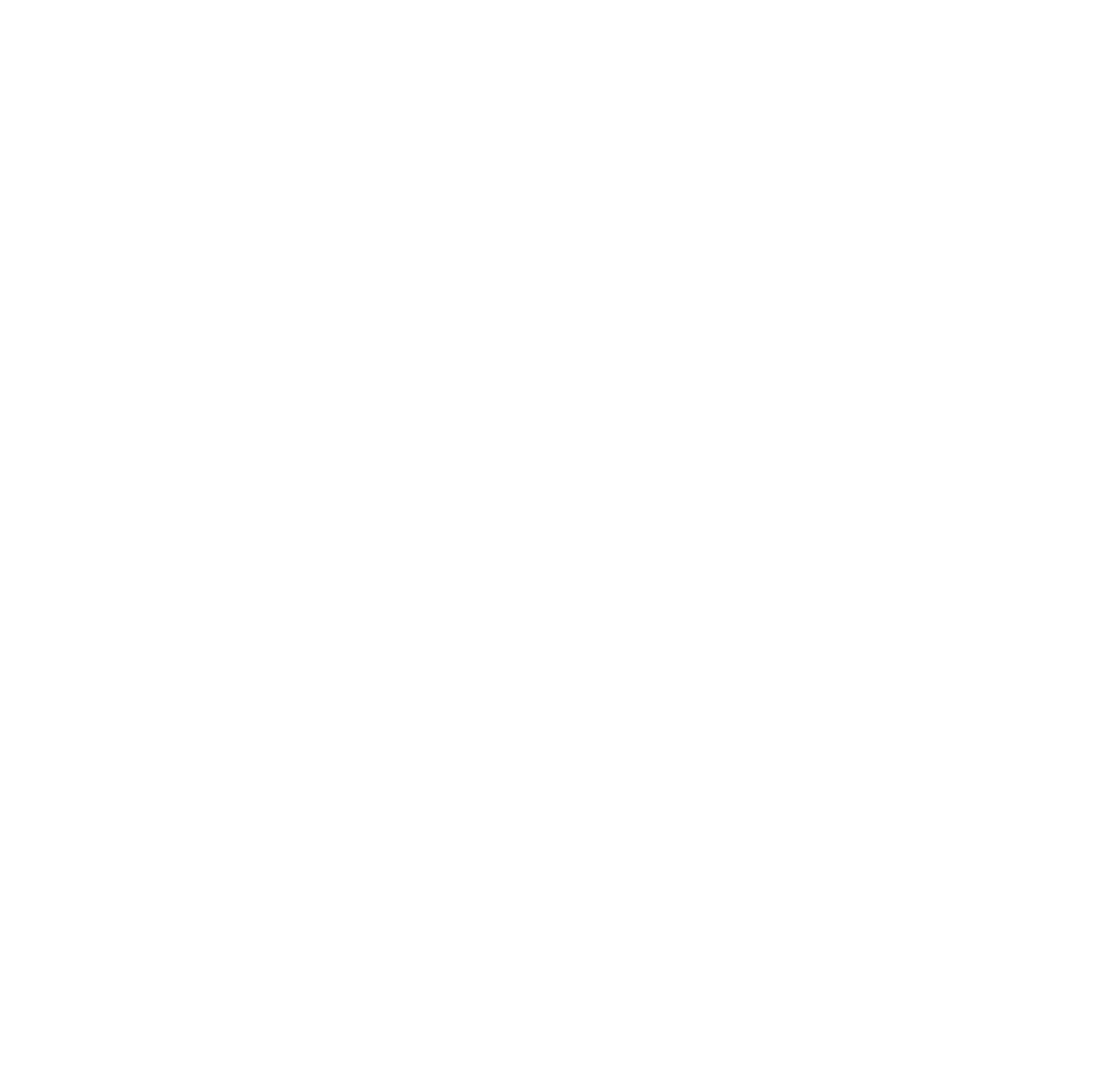

